\title{
Combination chemotherapy with irinotecan and cisplatin in pretreated patients with unresectable or recurrent gastric cancer
}

\author{
Shinya Ueda, Shuichi Hironaka, Narikazu Boku, Akira Fukutomi, Takayuki Yoshino, \\ and Yusuke ONOZAwA
}

Division of Gastrointestinal Oncology, Shizuoka Cancer Center, 1007 Shimonagakubo, Nagaizumi-cho, Sunto-gun, Shizuoka 411-8777, Japan

\begin{abstract}
Background. The combination of irinotecan (CPT-11) and cisplatin (CDDP) is an active regimen for metastatic gastric cancer in the first-line setting. The objective of this retrospective study was to clarify its efficacy and safety in patients with prior chemotherapy for advanced or recurrent gastric cancer. Methods. Patients in the study fulfilled the following selection criteria: (1) histologically proven gastric cancer with metastatic lesions; (2) performance status of 2 or less; (3) age of 75 years or younger; (4) at least one prior chemotherapy regimen without CPT-11 or CDDP; (5) adequate bone marrow, liver, and kidney function; (6) normal cardiac function; (7) no other severe medical conditions; (8) no other active malignancy; and (9) the provision of written informed consent. The treatment consisted of CPT-11 (70 mg/m²) on day 1 and day 15 and CDDP $\left(80 \mathrm{mg} / \mathrm{m}^{2}\right)$ on day 1 ; repeated every 4 weeks.

Results. Thirty-two patients were recruited, and 28 were assessable for clinical response. There were eight partial responses, resulting in a response rate of $28 \%$. Median time to progression was 104 days (range, 24-863 days) and median overall survival time was 283 days from the initiation of this therapy. The incidences of grade 4 neutropenia, grade 3 or higher infection, and diarrhea were $69 \%, 9 \%$, and $3 \%$, respectively. Other adverse reactions were mild. No treatment-related deaths occurred.

Conclusion. A combination of CPT-11 and CDDP may be active and feasible for gastric cancer patients with prior chemotherapy. Further studies with larger numbers of patients are needed to clarify this regimen's significance in the secondline setting.
\end{abstract}

Key words CPT-11 · CDDP · Gastric cancer · Prior chemotherapy

\section{Introductions}

Gastric cancer remains one of the major causes of cancer deaths all over the world. In Japan, despite the

Offprint requests to: S. Hironaka

Received: December 12, 2005 / Accepted: April 18, 2006 remarkable improvement in survival through early detection and curative surgery, there were approximately 50000 deaths from gastric cancer in 1997. Unresectable advanced or recurrent gastric cancer still shows a poor prognosis.

Although chemotherapy for patients with advanced gastric cancer can be palliative, we have made an effort to advance this treatment modality for prolonging survival and improving quality of life. Compared with the best supportive care, combination chemotherapy in these patents has been proven to improve the quality of life and the overall survival in four small randomized trials [1-4]. Recently, several new agents have been developed for advanced gastric cancer, and some promising data have been reported with docetaxel $[5,6]$, paclitaxel [7], irinotecan hydrochloride (CPT-11) [8], and S-1 (tegafur-gimeracil-oteracil potassium) [9]. While a first-line chemotherapy standard has not yet been established, these agents encourage us to define a second-line chemotherapy after failure of the first-line chemotherapy. However, there are only a few reports of the use of second-line chemotherapy for gastric cancer, and no standard regimen has been established. Therefore, investigation of a second-line regimen is very important.

As 5-fluorouracil (5-FU)-based regimens have been widely accepted as a standard first-line regimen at present, non-5-FU regimens are candidates as secondline regimens. Among the new agents mentioned above, CPT-11 is one of the most promising [8]. Boku et al. [10] reported that the response rate for CPT-11 and CDDP as the first-line regimen for advanced gastric cancer was $59 \%$ and the median survival time was 322 days. Ajani et al. [11] reported that the response rate for CPT-11 and CDDP therapy for first-line treatment was $58 \%$ and the median survival was 270 days. Both reports suggest that this combination therapy may also be very active and promising after the failure of first-line 5-FU-based chemotherapy. 
The objective of this retrospective study was to clarify the efficacy and safety of CPT-11 and CDDP cisplatin therapy in patients with prior chemotherapy for advanced or recurrent gastric cancer.

\section{Patients and methods}

\section{Recruitment criteria}

The subjects were 32 patients who were treated with CPT-11 and CDDP between September 2002 and September 2004 at the Shizuoka Cancer Center, Shizuoka, Japan. Patients who were recruited into the study fulfilled the following inclusion criteria: (1) histologically proven gastric cancer with metastatic lesions; (2) Eastern Clinical Oncology Group performance status of 2 or less; (3) age of 75 years or younger; (4) at least one prior chemotherapy regimen without CPT-11 or CDDP; (5) adequate bone marrow (WBC count $\geq 4000$ / $\mu \mathrm{l}$ and platelet count $\geq 10000 / \mu \mathrm{l}$ ), liver (serum bilirubin level $\leq 1.5 \mathrm{mg} / \mathrm{dl}$ and serum transaminase level $\leq$ three times the upper limit of normal range), and renal function (serum creatinine level $\leq 1.5 \mathrm{mg} / \mathrm{dl}$, blood urea nitrogen level $\leq 25 \mathrm{mg} / \mathrm{dl}$ ); (6) normal cardiac function; (7) no other severe medical conditions; (8) no other active malignancy; and (9) the provision of written informed consent.

\section{Statement of patients' informed consent}

Patients and their families submitted written informed consent prior to entry into the study. The information supplied covered: (1) the name of the disease and condition; (2) method and contents of the treatment; (3) expected response and side effects; (4) other possible outcomes; (5) advantages and disadvantages; (6) agreement, denial, and retraction; and, (7) human rights protection.

\section{Treatment schedule}

CPT-11 $\left(70 \mathrm{mg} / \mathrm{m}^{2}\right)$ was administered by intravenous infusion for $90 \mathrm{~min}$ on day 1 ; this was followed by a $2-\mathrm{h}$ interval, after which an intravenous infusion of CDDP $\left(80 \mathrm{mg} / \mathrm{m}^{2}\right)$ was administered over $2 \mathrm{~h}$, with adequate hydration. The same dose of CPT-11 was administered on day 15. This treatment was repeated every 4 weeks until disease progression, patient refusal, or unacceptable adverse reactions. On day 15 , if the patient had a WBC count of $2800 / \mu \mathrm{l}$ or less or $12000 / \mu \mathrm{l}$ or more, a platelet count of $100000 / \mu$ l or less, diarrhea of grade 1 or higher, or an episode of infection, then CPT-11 on day 15 was postponed until recovery from these adverse reactions. If these adverse reactions continued beyond day 22, then the CPT-11 which should have been admin- istered on day 15 was skipped. If a hematological adverse reaction was grade 4 , or a nonhematological adverse reaction was grade 3 or higher, then CPT-11 on day 15 was skipped, and the subsequent dose of CPT-11 was reduced to $60 \mathrm{mg} / \mathrm{m}^{2}$. Granisetron was used routinely before the administration of CPT-11. Granulocyte colony-stimulating factor (G-CSF) was used when necessary.

\section{Evaluation}

Tumor measurements for response assessment were obtained every 1-2 months by computed tomography (CT). The objective response to chemotherapy in measurable lesions was evaluated by the Response Evaluation Criteria in Solid Tumors [12]. National Cancer Institute common toxicity criteria (NCI-CTC; version 2) were adopted to evaluate the adverse reactions. The survival time was calculated from the day of the initiation of treatment to the date of death or the last date of confirmation of alive (censored), by the Kaplan-Meier method.

\section{Patient characteristics}

Patient characteristics are listed in Table 1. The median age of all patients was 58 years (range, 37-75 years).

Table 1. Patient characteristics

\begin{tabular}{|c|c|}
\hline Characteristic & \\
\hline Age (years) & \\
\hline Median & 58 \\
\hline Range & $(37-75)$ \\
\hline Sex & \\
\hline Male & 26 \\
\hline Female & 6 \\
\hline PS & \\
\hline $0 / 1 / 2$ & $16 / 14 / 2$ \\
\hline Prior chemotherapy & \\
\hline One regimen & 27 \\
\hline Two regimens & 5 \\
\hline S-1 & 18 \\
\hline $\mathrm{MTX}+5-\mathrm{FU}$ & 5 \\
\hline 5'-FUDR & 2 \\
\hline TXL & 3 \\
\hline UFT & 1 \\
\hline $5-\mathrm{FU}$ & 8 \\
\hline Histology & \\
\hline Intestinal & 16 \\
\hline Diffuse & 13 \\
\hline Unknown & 3 \\
\hline Metastatic site & \\
\hline Liver & 8 \\
\hline Lymph node & 23 \\
\hline Peritoneal dissemination & 13 \\
\hline Lung & 4 \\
\hline Ascites & 9 \\
\hline
\end{tabular}

PS, performance status; S-1, tegafur-gimeracil-oteracil-potassium; MTX, methotrexate; 5-FU, 5-fluorouracil; 5'-FUDR, doxifluridine; TXL, paclitaxel; UFT, uracil-futrafur 
Thirty patients $(94 \%)$ had a performance status of 0 or 1. Histologically, 16 patients $(50 \%)$ had an intestinal type of adenocarcinoma, 13 patients $(41 \%)$ had diffusetype gastric cancer, and 3 patients $(10 \%)$, had adenocarcinoma with unknown differentiation. Eighteen patients $(56 \%)$ had primary diseases and 28 patients $(88 \%)$ had measurable metastatic lesions. There were 8 patients $(25 \%)$ with metastases in the liver, $23(72 \%)$ with metastases in lymph nodes, $4(13 \%)$ with metastases in lung, 13 (41\%) with peritoneal dissemination, and $9(28 \%)$ with ascites. Prior chemotherapy regimens are listed in Table 1, and all patients showed progressive disease before the initiation of the regimen used in the present study.

\section{Results}

\section{Response and survival}

Twenty-eight patients with measurable metastatic lesions were assessable for clinical response. There were eight partial responses, resulting in an overall response rate of $28 \%$ (Table 2). Median time to progression was 104 days (range, 24-863 days) and median overall survival time was 283 days, with a median follow-up time of 345 days (Figs. 1, 2).

\section{Adverse reactions}

The total number of treatment courses was 109, and the median number in each patient was 2.5 courses (range, 1-6 courses). The adverse reactions to this regimen are summarized in Table 3 . The most frequent reaction

Table 2. Response

\begin{tabular}{ccccccc}
\hline & $n$ & CR & PR & SD & PD & RR \\
\hline Overall & 28 & 0 & 8 & 10 & 10 & $28 \%$
\end{tabular}

$\mathrm{CR}$, complete response; $\mathrm{PR}$, partial response; $\mathrm{SD}$, stable disease; PD, progressive disease; $\mathrm{RR}$, response rate was neutropenia, with grade 4 neutropenia observed in 22 patients $(69 \%)$, and grade 3 or higher infection observed in only 3 patients (9\%). Other hematologic adverse reactions were mild. Grade 3 diarrhea was observed in 1 patient (3\%). Other grade 3 or 4

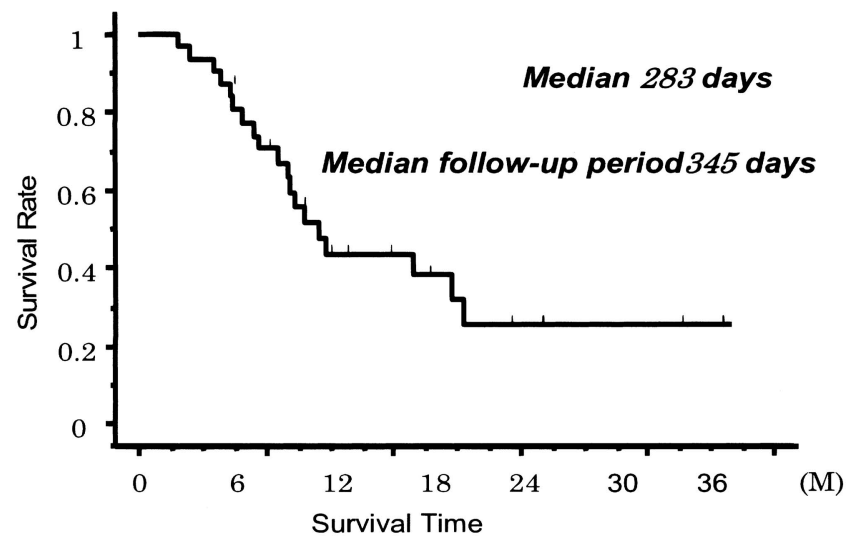

Fig. 1. Overall survival curve. Median overall survival time was 283 days. Median follow-up period was 345 days. The initial date of reckoning was the first day of the irinotecan (CPT-11), cisplatin (CDDP) chemotherapy

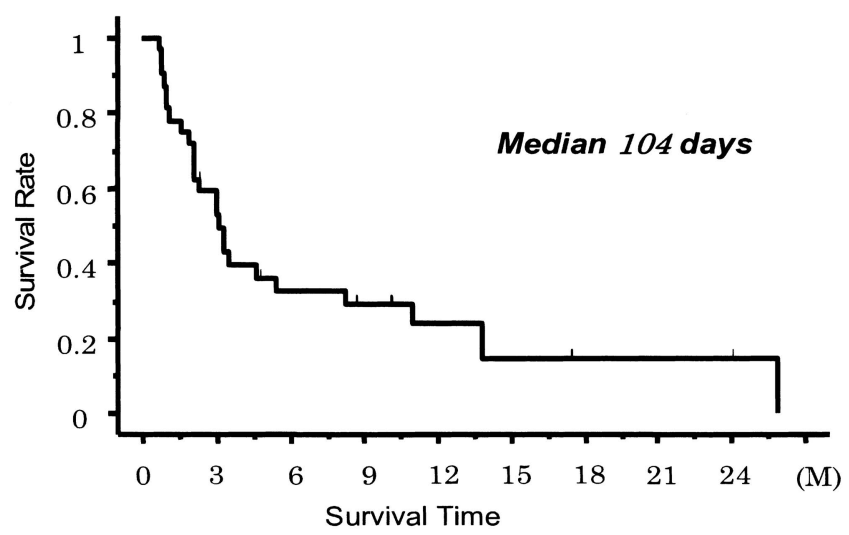

Fig. 2. Progression-free survival curve. Median time to progression was 104 days

Table 3. Adverse reactions

\begin{tabular}{lrrrcr}
\hline Grade & 1 & 2 & 3 & $4(\%)$ & $\begin{array}{c}\text { Percentage of patients } \\
\text { with grade } 3 \text { or higher }\end{array}$ \\
\hline Leukopenia & 0 & 6 & 17 & $7(22 \%)$ & $75 \%$ \\
Neutropenia & 0 & 4 & 4 & $22(69 \%)$ & $81 \%$ \\
Thrombocytopenia & 5 & 4 & 2 & 0 & $6 \%$ \\
Anemia & 8 & 3 & 9 & $5(16 \%)$ & $44 \%$ \\
Nausea & 11 & 13 & 7 & - & $22 \%$ \\
Vomiting & 7 & 7 & 4 & 2 & $19 \%$ \\
Anorexia & 11 & 13 & 4 & 2 & $3 \%$ \\
Diarrhea & 5 & 7 & 1 & 0 & $9 \%$ \\
Neutropenic fever & - & - & 3 & 0 & $0 \%$ \\
Creatinine & 8 & 7 & 0 & 0 & $3 \%$ \\
\hline
\end{tabular}


Table 4. Further chemotherapy

\begin{tabular}{lc}
\hline & No. of patients \\
\hline Further chemotherapy & \\
Yes & 26 \\
No & 5 \\
No follow-up & 1 \\
Treatment type & \\
TXL & 17 \\
CPT-11 & 6 \\
CDDPip & 2 \\
5-FU+LV & 1
\end{tabular}

TXL, paclitaxel; CPT-11, irinotecan; CDDPip, cispatin intraperitoneal; 5-FU, 5-fluorouracil; LV, leucovorin

nonhematological toxicities were: nausea, in 7 patients $(22 \%)$; vomiting, in 6 patients $(19 \%)$; and anorexia, in 6 patients $(19 \%)$. No patient was taken off treatment because of severe adverse reactions, and no treatmentrelated deaths occurred.

\section{Dose intensity}

The planned administration of CPT-11 on day 15 was not given $27(25 \%)$ times in 15 patients $(47 \%)$ during the 109 courses. Dose reduction of CPT-11 was required in $7(22 \%)$ patients. Leukopenia was the most common reason for skipping the administration of CPT-11 on day 15 and for the dose reduction of CPT-11 (days 1 and 15). Thus, the dose intensity of CPT- 11 was $24.5 \mathrm{mg} / \mathrm{m}^{2}$ per week and that of CDDP was $15.4 \mathrm{mg} / \mathrm{m}^{2}$ per week, which corresponded to $70 \%$ and $77 \%$ of the planned doses, respectively.

\section{Further chemotherapy}

Twenty-six patients $(81 \%)$ received further chemotherapy after failure of the CPT-11 + CDDP regimen (Table 4). The condition of the remaining 6 patients made them unsuitable to receive third-line chemotherapy.

\section{Discussion}

Because the administration of CPT-11 is contraindicated for patients with myelosupression, infection, diarrhea, ileus, interstitial pneumonia, and obstructive jaundice, for fear of its severe toxicity, in clinical practice we selected patients with good PS and mild peritoneal dissemination for second-line chemotherapy with CPT-11. Of all the patients, $30(94 \%)$ had a PS of 0 or 1 , $13(41 \%)$ had peritoneal dissemination, and $9(28 \%)$ had ascites; however, none had massive ascites. Thus, this study contained a certain level of selection bias.
In our study, the actual dose intensity of CPT-11 was $70 \%$ of the planned dose, which was lower than that in a previous report $(81.4 \%$ [10]). Leukopenia was the most common reason for skipping the administration of CPT-11 on day 15 , and for the dose reduction of CPT11. The incidences of grade 4 neutropenia (69\%) and grade 3 or 4 infection (9\%) were higher than those in the previous report [10], showing $57 \%$ and $5 \%$, respectively. The high incidence of neutropenia in our study may have been caused by the prior chemotherapy. On the other hand, the incidence of grade 3 or 4 diarrhea $(3 \%)$ was lower than that in the previous report $(20 \%$ [10]), and other toxicities were mild, with frequencies similar to those in the previous report. Although dose reduction was required in some of our selected patients, this regimen is considered to be feasible for patients with prior chemotherapy.

CPT-11 has activity against gastric carcinoma. When used as a single agent for untreated and treated gastric cancer patients, Kambe et al. [13] reported a response rate of $23 \%$. A recent European study of single-agent CPT-11 therapy reported a response rate of $17 \%$ in 34 previously untreated patients with gastric cancer [14]. There are a few reports of CPT-11 used in second-line combination chemotherapy regimens. Boku et al. [10] reported that CPT-11 and CDDP showed a response rate of $27 \%$. In a report by Ajani et al. [15], this combination showed a response rate of $31 \%$, with a median survival of 150 days. In a phase II study of CPT-11 and mitomycin C (MMC) [16], the response rate was $29 \%$, and the median survival was 306 days. Kim et al. [17] reported that CPT-11, 5-FU, and leucovorin showed a response rate of $21 \%$, and the median survival was 273 days. From the results of these studies, it seems that combination chemotherapy with CPT-11 may have a slightly higher response rate in second-line chemotherapy for gastric cancer than CPT11 alone.

Both paclitaxel and docetaxel have been widely used in second-line chemotherapy for gastric cancer. Arai et al. [18] reported that the response rate to paclitaxel was $23 \%$, and the median survival was 207 days. Park et al. [19] reported that combination chemotherapy with docetaxel and CCDP showed a response rate of $17 \%$, with a median survival of 174 days. These results seem to be comparable with those of CPT-11-containing chemotherapy as a second-line chemotherapeutic regimen for gastric cancer.

In conclusion, the combination of CPT-11 and CDDP may be active and feasible for patients with prior chemotherapy for gastric cancer. To establish standard second-line chemotherapy for gastric cancer, especially after the failure of a 5-FU-based regimen, a CPT-11based regimen and a taxane-based regimen should be compared in a randomized study. 


\section{References}

1. Murad AM, Santiago FF, Petroianu A, Rocha PR, Rodrigues MA, Rausch M. Modified therapy with 5-fluorouracil, doxorubicin, and methotrexate in advanced gastric cancer. Cancer 1993;72: 37-41.

2. Pyrhonen S, Kuitunen T, Kouri M. Randomised comparison of fluorouracil, epidoxorubicin and methotrexate (FEMTX) plus supportive care with supportive care alone in patients with nonresectable gastric cancer. Br J Cancer 1995;71:587-91.

3. Glimelius B, Hoffmann K, Haglund U, Nyren O, Sjoden PO. Initial or delayed chemotherapy with best supportive care in advanced gastric cancer. Ann Oncol 1994;5:189-90.

4. Scheithauer W, Komek G, Zeh B, et al. Palliative chemotherapy versus supportive care in patients with metastatic gastric cancer. A randomized trial (abstract). Second International Conference on Biology, Prevention, and Treatment of GI Malignancy, Koln, Germany; 1995. p. 68.

5. Taguchi T, Sakata Y, Kanamaru R, Kurihara M, Suminaga M, Ota J, et al. Late phase II clinical study of RP56976 (Docetaxel) in patients with advanced/recurrent gastric cancer: a Japanese Cooperative Study Group trial (Group A). Jpn J Cancer Chemother 1998;25:1915-24.

6. Mai M, Sakata Y, Kanamaru R, Kurihara M, Suminaga M, Ota J, et al. A late phase II clinical study of RP56976 (Docetaxel) in patients with advanced or recurrent gastric cancer: a Japanese Cooperative Study Group trial (Group B). Jpn J Cancer Chemother 1999;26:1487-96.

7. Hyodo I, Ohtsu A, Shirao K, Miyata Y, Saito H, Taguchi T. Late phase II study of paclitaxel (BMS-181339) in patients with advanced/recurrent gastric cancer; a Japanese Cooperative Study Group trial (group A). The $38^{\text {th }}$ annual meeting of Japan Society for Cancer Therapy 2002;35:443.

8. Futatsuki K, Wakui A, Nakao I, Sakata Y, Kambe M, Shimada Y, et al. Late phase II study of irinotecan hydrochloride (CPT-11) in advanced gastric cancer. Jpn J Cancer Chemother 1994;21:103338

9. Sakata Y, Ohtsu A, Horikoshi N, Sugimachi K, Mitachi Y, Taguchi T. Late phase II study of novel oral fluoropyrumidine anticancer drug S-1 (1 M tegafur- $0.4 \mathrm{M}$ gimestat- $1 \mathrm{M}$ otastat potassium) in advanced gastric cancer patients. Eur J Cancer 1998;34:1715-20.

10. Boku N, Ohtsu A, Shimada Y, Shirao K, Seki S, Saito H, et al. Phase II study of a combination of irinotecan and cisplatin against metastatic gastric cancer. J Clin Oncol 1999;17:319-23.

11. Ajani JA, Baker J, Pisters PW, Ho L, Mansfield PF, Feig BW, et al. CPT-11 plus cisplatin in patients with advanced, untreated gastric or gastroesophageal junction carcinoma. Cancer 2002;94: 641-6.

12. World Health Organization. WHO handbook for reporting results of cancer treatment (WHO Offset Publication No. 48). Geneva: World Health Organization; 1979.

13. Kambe M, Wakui A, Nakao I, et al. A late phase II study of irinotecan (CPT-11) in patients with advanced gastric cancer. Proc Am Soc Clin Oncol 1993;12:198.

14. Kohne CH, Thuss-Patience P, Catane R, Klein H, Peretz T, Preusser P, et al. Final results of a phase II trial of CPT-11 in patients with advanced gastric cancer (abstract). Proc Am Soc Clin Oncol 1999;18:258a.

15. Ajani JA, Baker J, Pisters PW, Ho L, Mansfield PF, Feig BW, et al. Irinotecan/cisplatin in advanced, treated gastric or gastroesophageal junction carcinoma. Oncol 2002;16:16-18.

16. Hamaguchi T, Ohtsu A, Hyodo I, Arai Y, Takiuchi H, Fujii H, et al. A phase II study of biweekly irinotecan and mitomycin $\mathrm{C}$ combination therapy in patients with fluoropyrimidine-resistant advanced gastric cancer: The Japan Clinical Oncology Group trial (JCOG0109). Proc Am Soc Clin Oncol 2004;22:4071.

17. Kim ST, Kang WK, Kang JH, Park KW, Lee J, Lee SH, et al. Salvage chemotherapy with irinotecan, 5-fluorouracil and leucovorin for taxane- and cisplatin-refractory, metastatic gastric cancer. Br J Cancer 2005;92:1850-4.

18. Arai T, Hamaguchi T, Shirao K, Shimada Y, Yamada Y, Muro K, et al. Weekly paclitaxel in patients with heavily treated advanced pastric cancer: Proc Am Soc Clin Oncol 2003;22:321.

19. Park SH, Kang WK, Lee HR, Park J, Lee KE, Lee SH, et al. Docetaxel plus cisplatin as second-line therapy in metastatic or recurrent advanced gastric cancer progressing on 5-fluorouracilbased regimen. Am J Clin Oncol 2004;27:477-80. 